\title{
The Relationship Between Subjective Socioeconomic Status and Job-Seeking Self-Efficacy: The Mediating Role of Pressure
}

\author{
Yang $\mathrm{Li}^{1,2}$, Tinghu Kang ${ }^{3, *}$ and Jiandong $\mathrm{Guo}^{4}$ \\ ${ }^{1}$ College of Education, Northwestern Normal University, Lanzhou, 730070, China \\ ${ }^{2}$ School of Teacher Education, Yuxi Normal University, Yuxi, 653100, China \\ ${ }^{3}$ School of Psychology, Northwestern Normal University, Lanzhou, 730070, China \\ ${ }^{4}$ Department of Admissions and Employment Service, Northwestern Normal University, Lanzhou, 730070, China \\ *Corresponding author: kangyan313@nwnu.edu.cn
}

\begin{abstract}
The purpose of this work was to explore the relationship between subjective socioeconomic status, pressure, and job-seeking self-efficacy, which adopted subjective socioeconomic status questionnaire, self-efficacy questionnaire and pressure perception questionnaire to investigate 771 college graduates. The results showed that the subjective economic status of college students has a positive predictive effect on job-seeking self-efficacy, and the pressure mediated the predictive effect. The mediating effect reveals the mechanism between the subjective socioeconomic status, pressure and job-seeking self-efficacy of college students, which is of great significance to enrich the employment education theory of college students with difficult employment and better carry out the employment guidance work of college students.

Keywords: Subjective socioeconomic status; Pressure; Job-seeking self-efficacy; Employment guidance
\end{abstract}

\section{INTRODUCTION}

The employment problem of college students is a major issue related to the livelihood and people's livelihood. The successful employment of college students is not only conducive to their own healthy development, but also conducive to social harmony and stability [1]. With the increasing number of college graduates and the increasingly fierce competition for employment, some college students have psychological problems such as depression, anxiety and even suicidal tendency because of excessive pressure, which will not only affect their mental health level, but also lead to the success or failure of job-seeking. At the same time, due to the downward pressure of the global economy, the transformation and development of China's industrial structure and the superimposed effects of COVID-19 epidemic, it is still difficult to change the graduate employment dilemma in the future and even in a longer period.

\section{LITERATURE REVIEW AND RESEARCH HYPOTHESIS}

\subsection{Literature Review}

Socioeconomic status is the judgment of the status of an individual or group in society based on the social resources they own [2]. Studies have shown that socioeconomic status has a significant correlation with students' academic engagement and self-efficacy [3-4]. The above studies generally use the method of weighted measurement of students' family income and parents' occupation to define students' objective family socioeconomic status, and replace students' individual socioeconomic status. However, the accuracy of self-reporting of family income and other information is debatable, and the perception of the objective socioeconomic status of their families may be inconsistent with the actual situation. Glendinning (1992) also pointed out that college students have gradually formed their own sense of social status, and objective indicators of family socioeconomic status can no longer replace their socioeconomic status [5]. Subjective socioeconomic status refers to an individual's perceived position in the social hierarchy [6], which has a more direct impact on an individual than objective socioeconomic status [7].

Job-seeking pressure, in the psychological sense, can be understood as the physiological, psychological and 
behavioral stress process caused by internal or external pressure source in the process of job-seeking [8]. Studies have shown that too much pressure will harm the mental health of job seekers, and there is a significant negative correlation between pressure and self-efficacy.

Job-seeking self-efficacy, that is, the degree of confidence that job seekers can successfully complete a series of job-seeking activities [11]. According to the research of Moynihan et al. (2003), individuals with high job-seeking self-efficacy have higher job-seeking success efficiency [12]. There are also empirical studies that show that self-efficacy plays a key role in successful employment of college students and mainly in self-cognition, career choice and career decision-making [13].

At present, there are few studies on how subjective socioeconomic status affects self-efficacy, and researchers pay less attention to the problem of job search of college students with low subjective socioeconomic status. The purpose of this work was to explore the mechanism between subjective socioeconomic status, pressure and self-efficacy of job-seeking, and provide theoretical and empirical basis for college students' employment guidance.

\subsection{Research Hypotheses}

To sum up, in order to examine the relationship between college students' subjective socioeconomic status and job-seeking self-efficacy, as well as the role of pressure in this relationship, a mediation model was constructed (see Fig. 1) and two hypotheses were proposed:

H1: The subjective socioeconomic status of college students has a negative predictive effect on job-seeking self-efficacy;

H2: Pressure plays a mediating role in the effect of subjective socioeconomic status on job-seeking self-efficacy.

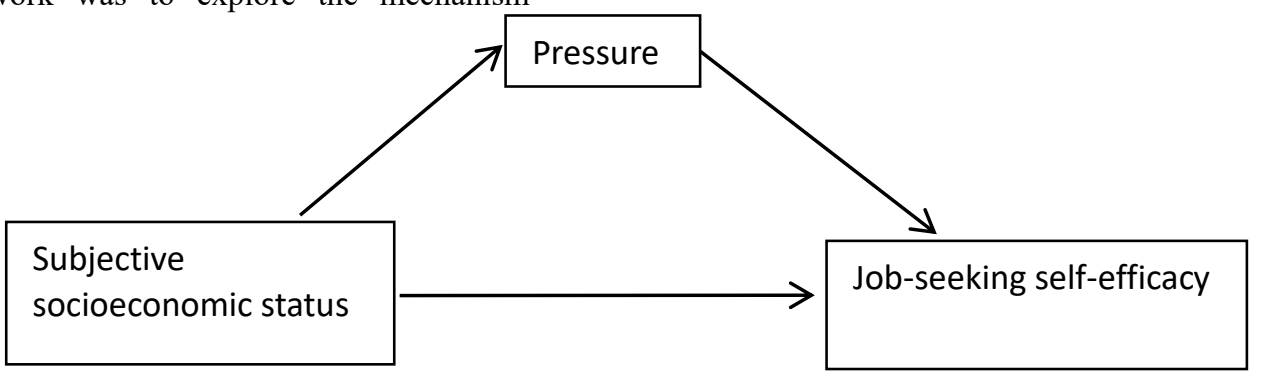

Figure 1. Hypothesis model

\section{RESEARCH METHOD}

\subsection{Research Subjects}

In this study, 771 valid questionnaires were obtained from senior undergraduates as subjects. The average age of the subjects is 21.9 years $(\mathrm{SD}=1.15)$. Among them, 335 are male, accounting for $43.5 \%$; 436 are female, accounting for $56.5 \%$; 650 are Han nationality, accounting for $84.3 \%$; 121 are ethnic minorities, accounting for $15.7 \%$; 469 are from countryside, accounting for $60.8 \%$, and 302 are from urban, accounting for $39.2 \%$.

\subsection{Research Tools}

\subsubsection{Subjective socioeconomic status}

MacArthur Scale of Subjective Social Status compiled by Adler is adopted [14], which has two topics, each with a score of 10 . The higher the total score, the higher the subjective socioeconomic status of the individual. And the $\alpha$ coefficient of the scale in this work is 0.79 .

\subsubsection{Pressure}

The Chinese version of PSS-10 scale (Liu Wanting et al., 2015) [15] was adopted, with a total of 10 items, including two dimensions of crisis perception and coping ability. Likert5 points are used to score, including 4 reverse scoring questions. The higher the score is, the higher the pressure perception is. The $\alpha$ coefficient of the scale in this work is 0.76 .

\subsubsection{Job-seeking self-efficacy.}

The Job-seeking Self-efficacy Scale [16] compiled by Hu Yanhong et al. (2016) was adopted, and it contains 17 items, including three dimensions of vocational competence, career orientation and career information search. By using Likert5 points, the sum of the three dimensions is the overall evaluation score of job-seeking self-efficacy. The higher the score, the more positive the evaluation of job-seeking self-efficacy, and the $\alpha$ coefficient of the scale in this work is 0.95 . 


\subsection{Data Processing and Common Method Deviation Test}

SPSSPROCESS macroprogram is used to analyze the mediating effect of the data, and the effect test is carried out by constructing 5000 random samples with replacements and estimating $95 \%$ confidence intervals. Harman single-factor test is used to test the common method bias for the collected data. A total of 5 factors with a characteristic root greater than 1 are extracted from the unrotated exploratory factor analysis results, among which the interpretation rate of the maximum factor variance is less than $40 \%$, which proves that there is no serious common method bias in this work.

\section{DATA ANALYSIS AND RESULTS}

\subsection{Descriptive Statistical Results of Variables}

Descriptive statistics and correlation analysis are conducted on all variables in this work.

Table 1. Descriptive statistics and correlation analysis of each variable $(n=771)$

\begin{tabular}{cccccccc}
\hline Variable & M & SD & $\mathbf{1}$ & $\mathbf{2}$ & $\mathbf{3}$ & $\mathbf{4}$ & $\mathbf{5}$ \\
\hline 1. Gender & 1.57 & 0.50 & 1 & & & & \\
2. Origin & 1.39 & 0.49 & -0.063 & 1 & & & \\
3. Subjective socioeconomic status & 8.31 & 3.21 & 0.047 & $0.281^{* *}$ & 1 & & \\
4. Pressure & 19.50 & 4.44 & $0.091^{*}$ & $-.100^{* *}$ & $-.258^{* *}$ & 1 & \\
5. Job-seeking self-efficacy & 55.92 & 8.72 & $-.129^{* *}$ & $0.086^{*}$ & $0.307^{* *}$ & $-.321^{* *}$ & 1 \\
\hline
\end{tabular}

Table 1 shows that the job-seeking self-efficacy is significantly negatively correlated with subjective socioeconomic status and positively correlated with pressure, which indicates that college students with low subjective socioeconomic status have a low level of job-seeking self-efficacy, and college students with high pressure level also have a low level of job-seeking self-efficacy. There is a significant negative correlation between the origin of students and the pressure, and a significant positive correlation with the subjective socioeconomic status and the job-seeking self-efficacy, which shows that compared with urban students, college students from rural areas have a higher level of pressure and a lower subjective socioeconomic status and job-seeking self-efficacy. In terms of gender differences, the pressure level of female students is higher than that of male students, while the job-seeking self-efficacy is lower than that of male students.

\subsection{Relationship between Subjective Socioeconomic Status and Job-Seeking Self-Efficacy: The Mediating Role of Pressure}

First, Mode 14 (a simple mediation model) in the Process macroprogram compiled by Hayes (2012) is adopted to test the mediating effect of pressure on the relationship between subjective socioeconomic status and job-seeking self-efficacy. The results (see Table 2) show that subjective socioeconomic status has a significant positive predictive effect on job-seeking self-efficacy $(B=0.83, t=8.59$, $\mathrm{p}<0.01)$. The test results of the mediating effect of pressure on the relationship between subjective socioeconomic status and job-seeking self-efficacy (see Table 2) show that when the mediating variable (pressure) is included, the direct positive predictive effect of subjective socioeconomic status on job seeking self-efficacy is still significant $(\mathrm{B}=0.66, \mathrm{t}=6.82, \mathrm{p}<0.01)$.

Moreover, the Bootstrap test results find that the upper and lower limits of the Bootstrap 95\% confidence interval between the direct effect of subjective socioeconomic status on job-seeking self-efficacy and the stress-mediated effect do not contain 0 , which indicates that subjective socioeconomic status can not only directly predict job-seeking self-efficacy, but also predict job-seeking self-efficacy through the mediating effect of pressure. The proportion of direct effect and intermediary effect in total effect is shown in Table 3 . 
Table 2. Mediation Model Test of Pressure

\begin{tabular}{ccccccc}
\hline \multicolumn{2}{c}{ Regression equation } & \multicolumn{2}{c}{$\begin{array}{c}\text { Overall fitting } \\
\text { index }\end{array}$} & \multicolumn{2}{c}{$\begin{array}{c}\text { Significance of regression } \\
\text { coefficient }\end{array}$} \\
\hline Result variables & Predicted variables & $\mathbf{R}$ & $\mathbf{R}^{2}$ & $\mathbf{F}$ & $\mathbf{B}$ & t \\
\hline Job-seeking self-efficacy & Subjective socioeconomic status & & & & 0.83 & $8.59^{* *}$ \\
& Age & 0.31 & 0.09 & $26.67^{* *}$ & 0.06 & 0.22 \\
& Origin & & & & 0.01 & 0.02 \\
\hline Pressure & Subjective socioeconomic status & & & & -0.35 & $-6.87^{* *}$ \\
& Age & 0.26 & 0.07 & $18.51^{* *}$ & -0.02 & -0.13 \\
& Origin & & & & -0.28 & -0.84 \\
\hline Self-efficacy & Subjective socioeconomic status & & & & 0.66 & $6.82^{* *}$ \\
& Pressure & 0.40 & 0.16 & $35.65^{* *}$ & -0.51 & $-7.54^{* *}$ \\
& Age & & & & -0.05 & 0.19 \\
& Origin & & & -0.21 \\
\hline
\end{tabular}

Table 3. The mediating effect of pressure on the relationship between subjective socioeconomic status and job-seeking self-efficacy

\begin{tabular}{cccccc}
\hline & Effect & BootSE & BootLLCI & BootULCI & Relative effect value \\
\hline Total effects & 0.83 & 0.07 & -0.64 & -0.38 & \\
Indirect effects & 0.18 & 0.04 & 0.10 & 0.26 & $21.02 \%$ \\
Direct effects & 0.66 & 0.09 & 0.48 & 0.84 & $78.98 \%$ \\
\hline
\end{tabular}

\section{CONCLUSION AND RECOMMENDATIONS}

The results of work study show that both hypothesis H1 and $\mathrm{H} 2$ are verified. The subjective socioeconomic status of college students has a negative predictive effect on job-seeking self-efficacy, and pressure plays a mediating role in the influence of subjective socioeconomic status on job-seeking self-efficacy. In view of this research result, promoting the high-quality employment of college students can start from the following aspects:

\subsection{Enhancing the Job-Seeking Self-Efficacy of College Students}

Colleges and universities need to strengthen the practicality of employment guidance courses, enhance students' practical ability to seek employment, enhance their job-seeking competence, and clarify their job-seeking goals. Teachers should teach students the necessary methods of searching and identifying information (how to find effective information, how to identify information authenticity and enterprise qualification in mass information), and improve their job-seeking self-efficacy. In addition, college students themselves also need to know themselves deeply, explore their own potential, improve their ability in all aspects, obtain employment smoothly and adapt to the needs of career development.

\subsection{Guiding College Students to Relieve Their Employment Pressure}

This work found that college students with low subjective socioeconomic status also have a low sense of job-seeking self-efficacy, which will further lead to a decrease in job-seeking self-efficacy when they are under greater pressure in the process of job-seeking. This low-confidence and high-pressure state are also easy to cause anxiety, depression and other psychological problems. Therefore, it is an effective way to relieve the employment pressure of college students and improve their sense of self-efficacy. Some studies have shown that emotional regulation can improve college students' employability, and this ability of emotion regulation can be intervened through teaching [18]. In view of the employment pressure of graduates, colleges and universities can help students master effective methods of regulating bad emotions, relieve the pressure of employment, and face the job search with a more positive attitude by designing training courses based on relevant psychological theories and teaching relevant skills for college students.

\subsection{Increasing Employment Guidance and Policy Support for the Groups with Employment Difficulty}

At present, the Ministry of Education and colleges and universities have issued relevant policies to provide employability improvement training, job recommendation and other employment assistance to graduates from 
registered low-income families, physical disabilities and other groups with employment difficulties. However, there is a general lack of attention to the group of college students with low subjective economic status. College students whose family income is slightly higher than the poverty level are still in a weak position in the living environment, and their life is still difficult, thus they have negative evaluation and judgment on themselves. Therefore, relevant departments should formulate a more reasonable classification standard for groups with employment difficulties and provide necessary policy support and guidance to college students with low subjective economic status.

In short, improving college students' job-seeking self-efficacy is an effective way to promote their high-quality employment. Families and society also need to pay more attention to the groups with low job-seeking self-efficacy, so as to form the trinity force of "family", "school" and "community" to support and guide them to successful employment. Simultaneously, it is necessary to pay attention to the influence of pressure and other bad emotions on college students' sense of self-efficacy, and guide college students to manage their bad emotions well. Finally, it is necessary to accurately identify and help college students with low subjective socioeconomic status, carry out targeted early intervention on the negative impact of job-seeking self-efficacy, guide them to improve their abilities in all aspects, improve attribution methods, form a positive self-evaluation, and then promote their successful employment.

\section{REFERENCES}

[1] Zhang Yunlong, Ye Baojuan, Luo Zhaosheng et al. Effects of Coping Efficacy on College Students' Employability: The Mediating Role of Career Exploration [J]. Chinese Journal of Clinical Psychology, 2015, 23 (05): 815-817.

[2] Bradley, Robert H.; Corwyn, Robert F. Socioeconomic Status and Child Development [J]. Annual Review of Psychology, 2002, 53 (1): 371-399.

[3] Shi Leishan, Chen Yingmin, Hou Xiu, Gao Fengqiang. The Relationship between Family Socioeconomic Status and Learning Engagement: The Mediating Role of Academic Self-efficacy [J]. Psychological Development and Education, 2013, 29 (01): 71-78.

[4] Chu Xiaoyuan, Liyuan, Wang Xingchao, Wang Pengcheng, Lei Li. Relationship between Family Socioeconomic Status and Adolescent Self-efficacy: Intermediation of Family Support and Regulation of Gender Factors [J]. Psychological Science, 2019, 42 (04): 891-897.
[5] Glendinning A, Love, John G., Hendry, Leo B. Adolescence and Health Inequalities: Extensions to Macintyre and West [J]. Social Science \& Medicine, 1992 (5), 679-687.

[6] Jackman, M.R., \& Jackman, R.W. An Interpretation of the Relation between Objective and Subjective Social Status [J]. American Sociological Review, 1973, 38 (5), 569-582.

[7] Sakurai, K., Kawakami, N., Yamaoka, K., Ishikawa, H., \& Hashimoto, H. The Impact of Subjective and Objective Social Status on Psychological Distress Among Men and Women in Japan [J]. Social Science \& Medicine, 2010, 70 (11), 1832-1839.

[8] Liu Zhihan. Employment Pressure and Subjective Well-being of College Students: Two-way Mediating Effect [J]. Chinese Journal of Clinical Psychology, 2019, 27 (02): 378-382.

[9] Deng Xiaohong, Zhang Jie, Lu Yang et al. Relationship between Employment Pressure and Mental Health of Poor College Students: The Mediating of Coping Style $[\mathrm{J}]$. Educational Research and Experiment, 2015, (01): 86-91.

[10] Yuan Jinfang. Study on Career Efficiency and Employment Pressure of 2011 College Graduates [J]. Education and Vocation, 2012 (20): 88-89.

[11] Taylor KM, Betz NE. Applications of Self-efficacy Theory to the Understanding and Treatment of Career Indecision [J]. Journal of Vocation Behavior, 1983, 22 (1): 63-81.

[12] Lisa M. Moynihan, Mark V. Roehling, Marcie A. LePine, et al. A Longitudinal Study of the Relationships among Job Search Self-Efficacy, Job Interviews, and Employment Outcomes [J]. Journal of Business and Psychology, 2003, 18(2): 207-233.

[13] Wen Duohong. Differences and Cultivating Ways of College Students' Career Selection Efficacy [J]. Education and Vocation, 2010 (24): 86-87.

[14] Chen Yuning, Yao Shuqiao, Xia Liangwei. Validity and Reliability of the Chinese Version of the Subjective Socioeconomic Status Scale for the Evaluation of Adult Samples [J]. Chinese Mental Health Journal, 2014, 28 (11): 869-874.

[15] Liu Wanting, Yi Jinyao, Zhong Mingtian, Zhu Xiongzhao. Equivalence of Perceived Stress Scale in College Students of Different Genders [J]. Chinese Journal of Clinical Psychology, 2015, 23 (5): 944-946. 
[16] Hu Yanhong, Liu Xia. Comparative Study of College Students' Elective Sense of Career [J]. Journal of China Women's University, 2006, (02): 22-27.

[17] Dacre Pool, L., \& Qualter, P. Emotional Self-efficacy, Graduate Employability and Career Satisfaction: Testing the Associations [J]. Australian Journal of Psychology, 2013, 65(4): 214-223.

[18] Pool, L.D., \& Qualter, P. Improving Emotional Intelligence and Emotional Self-efficacy through a Teaching Intervention for College Students [J]. Learning and Individual Differences, 2012, 22(3): 306-312. 